\title{
EXPONENTIAL DICHOTOMIES FOR IMPULSIVE EQUATIONS VIA QUADRATIC FUNCTIONS
}

\author{
LUIS BARREIRA AND CLAUDIA VALLS
}

\begin{abstract}
We give a characterization of the existence of a nonuniform exponential dichotomy for a linear impulsive differential equation. The characterization uses quadratic functions and the symmetric matrices defining them. As an application, we give a simple proof of the robustness property of a nonuniform exponential dichotomy under sufficiently small linear perturbations.
\end{abstract}

1. Introduction. Impulsive differential equations yield a smooth evolution that at certain times may change abruptly, namely, consider a linear impulsive differential equation:

$$
x^{\prime}=A(t) x, \quad t \neq \tau_{i},\left.\quad \Delta x\right|_{t=\tau_{i}}=B_{i} x
$$

on a finite-dimensional space $\mathbb{R}^{p}$, where $A(t)$ and $B_{i}$ are $p \times p$ matrices, with $A(t)$ varying continuously with $t \in \mathbb{R}$. Sometimes $\tau_{i}$ the evolution is smooth and is determined by the differential equation $x^{\prime}=A(t) x$, while at other times $\tau_{i}$ there are jumps determined by the condition $\left.\Delta x\right|_{t=\tau_{i}}=B_{i} x$, that is,

$$
x\left(\tau_{i}^{+}\right)=x\left(\tau_{i}\right)+B_{i} x\left(\tau_{i}\right) .
$$

This gives rise to unique and global left-continuous solutions of equation (1.1). We refer the reader to $[\mathbf{9}, \mathbf{1 9}]$ for an extensive list of references and for descriptions of many applications of the theory.

Our main aim is to give a characterization of when equation (1.1) admits a nonuniform exponential dichotomy in terms of quadratic functions and the matrices defining them, following to the possible

2010 AMS Mathematics subject classification. Primary 34D09, 34D10.

Keywords and phrases. Lyapunov functions, nonuniform hyperbolicity, robustness.

Supported by Portuguese funds through FCT-Fundação para a Ciência e a Tecnologia, project No. PEst-OE/EEI/LA0009/2013 (CAMGSD).

Received by the editors on October 2, 2014, and in revised form on February 14, 2015 .

DOI: $10.1216 /$ RMJ-2016-46-6-1771

Copyright (C)2016 Rocky Mountain Mathematics Consortium 
extent the approach in [2] for nonimpulsive equations. We then use this characterization to establish in a very simple manner the robustness property of a nonuniform exponential dichotomy. This means that, if equation (1.1) admits a nonuniform exponential dichotomy, then for any sufficiently small $B(t)$ and $C_{i}$ in some appropriate class of matrices, the equation

$$
x^{\prime}=[A(t)+B(t)] x, \quad t \neq \tau_{i},\left.\quad \Delta x\right|_{t=\tau_{i}}=\left(B_{i}+C_{i}\right) x
$$

also admits a nonuniform exponential dichotomy.

We emphasize that the robustness property has already been obtained [3], although with a much more elaborate proof in terms of fixed points of appropriate contraction maps. Some of the arguments in [3] are inspired by the work of Popescu [18] for uniform exponential dichotomies. Here, we present a much simpler proof based on the characterization in terms of quadratic functions.

The main difference between a uniform exponential dichotomy and a nonuniform exponential dichotomy is that, although in both situations we have an exponential stability or instability of the solutions, respectively, along the stable and unstable directions, in the nonuniform case the stability may be nonuniform on the initial time. We emphasize that the notion of a nonuniform exponential dichotomy is much more common than its classical uniform counterpart. In particular, from the point of view of ergodic theory, almost all trajectories having nonzero Lyapunov exponents admit a nonuniform exponential dichotomy. More precisely, let

$$
x^{\prime}=F(x)
$$

be an autonomous differential equation on $\mathbb{R}^{p}$, and assume that $\operatorname{div} F$ $=0$. Moreover, consider the flow

$$
\varphi_{t}\left(x_{0}\right)=x\left(t, x_{0}\right), \quad t \in \mathbb{R},
$$

defined by the equation, where $x\left(\cdot, x_{0}\right)$ is the solution $x=x(t)$ of equation (1.3) with $x(0)=x_{0}$. Since $\operatorname{div} F=0$, the flow preserves volume, that is, for each $t \in \mathbb{R}$ and each measurable set $A \subset \mathbb{R}^{p}$ the volumes of the sets $A$ and $\varphi_{t}(A)$ are equal. Then, on any invariant subset of finite volume, one can show that, for almost all initial 
conditions $x_{0} \in \mathbb{R}^{p}$, the linear variational equation

$$
y^{\prime}=A_{x_{0}}(t) y, \quad \text { where } \quad A_{x_{0}}(t)=d_{\varphi_{t}\left(x_{0}\right)} F,
$$

admits a nonuniform exponential dichotomy whenever all of its Lyapunov exponents are nonzero. For example, this happens on any compact energy level of a Hamiltonian system,

$$
q^{\prime}=\frac{\partial H}{\partial p}, \quad p^{\prime}=-\frac{\partial H}{\partial q},
$$

taking the usual Liouville volume. In this context, our work can also be considered a contribution to the theory of nonuniform hyperbolicity. We refer the reader to [1] for a detailed exposition, which goes back to the landmark works of Oseledets [15], and particularly, Pesin [16]. It is an important part of the general theory of dynamical systems and a principal tool in the study of stochastic behavior.

Our work can also be seen as a development of classical approaches of Dalec $^{\prime}$ kiı̆ and Krĕ̌n [7, Chapter 2] and Massera and Schäffer [13, Chapter 9] for uniform exponential behavior. The use of Lyapunov functions in the study of the stability of solutions of differential equations goes back to the seminal work of Lyapunov in his 1892 thesis (see [11]). Among the first accounts of the theory are the books by LaSalle and Lefschetz [10], Hahn [8] and Bhatia and Szegö [4]. The study of robustness also has a long history. In particular, it was discussed by Massera and Schäffer [12], Coppel [6] and Dalec'kiŭ and Kreŭn [7] in the case of Banach spaces. For more recent work in the case of uniform exponential behavior we refer the reader to $[\mathbf{5}, \mathbf{1 4}, \mathbf{1 7}, \mathbf{1 8}]$ and the references therein.

2. Quadratic functions. Let $M_{p}$ be the set of all $p \times p$ matrices with real entries. We consider the linear impulsive differential equation (1.1) on $\mathbb{R}^{p}$, where $A(t)$ and $B_{i}$ are in $M_{p}$, with $A(t)$ varying continuously with $t \in \mathbb{R}$. Moreover, we assume that the jumping times

$$
\cdots<\tau_{-2}<\tau_{-1}<0<\tau_{1}<\tau_{2}<\cdots
$$

satisfy $\lim _{i \rightarrow \pm \infty} \tau_{i}= \pm \infty$ and

$$
q:=\sup _{t>\tau} \frac{\operatorname{card}\left\{i \in \mathbb{Z}: \tau \leq \tau_{i}<t\right\}}{1+t-\tau}<\infty .
$$


We note that there exist unique left-continuous solutions of equation (1.1) and that these are global (see, for example, [19]). We write each solution in the form $x(t)=T(t, s) x(s)$ for $t, s \in \mathbb{R}$, where $T(t, s)$ is the associated linear evolution operator. We note that

$$
T(t, s) T(s, r)=T(t, r) \quad \text { and } \quad T(t, t)=\mathrm{Id}
$$

for $t, s, r \in \mathbb{R}$, including when replaced, respectively, by $t^{+}, s^{+}$and $r^{+}$.

Given symmetric invertible matrices $S(t) \in M_{p}$ for $t \in \mathbb{R}$, we consider the function $H: \mathbb{R} \times \mathbb{R}^{p} \rightarrow \mathbb{R}$ defined by

$$
H(t, x)=\langle S(t) x, x\rangle .
$$

Moreover, we consider the class $\mathcal{D}$ of all left-continuous functions $F$ on $\mathbb{R}$ (with values on $\mathbb{R}$ or on $M_{p}$ ) at most with discontinuities at the jumping times $\tau_{i}$ such that, for each $i \in \mathbb{Z}$, there exists a $C^{1}$ extension $G_{i}$ of $F \mid\left(\tau_{i}, \tau_{i+1}\right)$ to an open interval containing $\left[\tau_{i}, \tau_{i+1}\right]$. For each function $F \in \mathcal{D}$, we define

$$
F^{\prime}\left(\tau_{i}\right)=\lim _{h \rightarrow 0^{-}} \frac{F\left(\tau_{i}+h\right)-F\left(\tau_{i}\right)}{h}=G_{i-1}\left(\tau_{i}\right)
$$

and

$$
F^{\prime}\left(\tau_{i}^{+}\right)=\lim _{h \rightarrow 0^{+}} \frac{F\left(\tau_{i}+h\right)-F\left(\tau_{i}^{+}\right)}{h}=G_{i}\left(\tau_{i}\right) .
$$

Assuming that $t \mapsto S(t)$ is in $\mathcal{D}$, using equations (2.2) and (2.3), one can define

$$
\dot{H}(t, x)=\left.\frac{d}{d h} H(t+h, T(t+h, t) x)\right|_{h=0}
$$

for $t \in \mathbb{R}$ (including for $t=\tau_{i}^{+}$) and $x \in \mathbb{R}^{p}$. In particular,

$$
\dot{H}\left(\tau_{i}, x\right)=\lim _{h \rightarrow 0^{-}} \frac{H\left(\tau_{i}+h, T\left(\tau_{i}+h, \tau_{i}\right) x\right)-H\left(\tau_{i}, x\right)}{h}
$$

and

$$
\dot{H}\left(\tau_{i}^{+}, x\right)=\lim _{h \rightarrow 0^{+}} \frac{H\left(\tau_{i}+h, T\left(\tau_{i}+h, \tau_{i}^{+}\right) x\right)-H\left(\tau_{i}^{+}, x\right)}{h} .
$$

For each $\tau \in \mathbb{R}$, let

$$
F_{\tau}^{s}=\left\{x \in \mathbb{R}^{p}: H(t, T(t, \tau) x) \geq 0 \quad \text { for } t \in \mathbb{R}\right\}
$$


and

$$
F_{\tau}^{u}=\left\{x \in \mathbb{R}^{p}: H(t, T(t, \tau) x) \leq 0 \quad \text { for } t \in \mathbb{R}\right\}
$$

Theorem 2.1. Assume that $t \mapsto H(t, x(t))$ is continuous and is in $\mathcal{D}$ for any solution $x(t)$ of equation (1.1). If there exists $\theta \in(0,1)$ such that

$$
\dot{H}(t, x) \leq \log \theta|H(t, x)|
$$

for every $t \in \mathbb{R}$ and $x \in \mathbb{R}^{p}$, then

$$
H(t, T(t, \tau) x) \leq \theta^{(t-\tau)} H(\tau, x)
$$

for $t \geq \tau$ and $x \in F_{\tau}^{s}$, and

$$
|H(t, T(t, \tau) x)| \geq \theta^{-(t-\tau)}|H(\tau, x)|
$$

for $t \geq \tau$ and $x \in F_{\tau}^{u}$.

Proof. We begin with an auxiliary result.

Lemma 2.2. Given a continuous function $w:[\tau, t] \rightarrow \mathbb{R}^{+}$and $a$ constant $M>0$, if

$$
w(\alpha)-w(\tau) \geq M \int_{\tau}^{\alpha} w(v) d v
$$

for $\alpha \in[\tau, t]$, then $w(\alpha) \geq w(\tau) e^{M(\alpha-\tau)}$ for $\alpha \in[\tau, t]$.

Proof of Lemma 2.2. By equation (2.11), we have

$$
\int_{\tau}^{\alpha} \frac{w(u)}{w(\tau)+M \int_{\tau}^{u} w(v) d v} d u \geq \alpha-\tau,
$$

and thus,

$$
\log \left(w(\tau)+M \int_{\tau}^{\alpha} w(v) d v\right)-\log w(\tau) \geq M(\alpha-\tau) .
$$

Finally, again by equation (2.11),

$$
w(\alpha) \geq w(\tau)+M \int_{\tau}^{\alpha} w(v) d v \geq w(\tau) e^{M(\alpha-\tau)},
$$

which yields the desired inequality. 
Take $x \in F_{\tau}^{u}$. We consider $t \geq \tau$ such that $[\tau, t]$ contains no jumping time. For $u \in[\tau, t]$ and $x(u)=T(u, \tau) x$, we obtain

$$
H(u, x(u))-H(\tau, x(\tau))=\int_{\tau}^{u} \dot{H}(v, x(v)) d v \leq \log \theta \int_{\tau}^{u}|H(v, x(v))| d v .
$$

Hence,

$$
|H(u, x(u))|-|H(\tau, x)| \geq \log (1 / \theta) \int_{\tau}^{u}|H(v, x(v))| d v,
$$

and it follows from Lemma 2.2 that

$$
|H(t, x(t))| \geq \theta^{-(t-\tau)}|H(\tau, x)| .
$$

This shows that inequality (2.10) holds when $[\tau, t]$ contains no jumping times. Otherwise, since $t \mapsto H(t, x(t))$ is continuous, it is sufficient to consider the case when $[s, t]$ contains a single jumping time $\tau_{i}$. It follows from equation (2.12) that

$$
|H(t, x(t))| \geq \theta^{-\left(t-\tau_{i}\right)}\left|H\left(\tau_{i}^{+}, x\left(\tau_{i}^{+}\right)\right)\right|,
$$

and, since $t \mapsto H(t, x(t))$ is continuous, we have $H\left(\tau_{i}^{+}, x\left(\tau_{i}^{+}\right)\right)=$ $H\left(\tau_{i}, x\left(\tau_{i}\right)\right)$. Moreover, on the interval $\left[\tau, \tau_{i}\right]$, it follows again from equation (2.12) that

$$
\left|H\left(\tau_{i}, x\left(\tau_{i}\right)\right)\right| \geq \theta^{-\left(\tau_{i}-\tau\right)}|H(\tau, x(\tau))|,
$$

and so,

$$
\begin{aligned}
|H(t, x(t))| & \geq \theta^{-\left(t-\tau_{i}\right)}\left|H\left(\tau_{i}^{+}, x\left(\tau_{i}^{+}\right)\right)\right| \\
& =\theta^{-\left(t-\tau_{i}\right)}\left|H\left(\tau_{i}, x\left(\tau_{i}\right)\right)\right| \\
& \geq \theta^{-\left(t-\tau_{i}\right)} \theta^{-\left(\tau_{i}-\tau\right)}|H(\tau, x)| \\
& =\theta^{-(t-\tau)}|H(\tau, x)|
\end{aligned}
$$

which establishes inequality (2.10).

Now take $x \in F_{\tau}^{s}$ and $t \geq \tau$ such that $[\tau, t]$ contains no jumping times. For $u \in[\tau, t]$ and $x(u)=T(u, \tau) x$, we obtain

$$
H(t, x(t))-H(u, x(u))=\int_{u}^{t} \dot{H}(v, x(v)) d v \leq \log \theta \int_{u}^{t} H(v, x(v)) d v .
$$


Consider the function $w:[\tau, t] \rightarrow \mathbb{R}^{+}$defined by

$$
w(z)=H(t+\tau-z, x(t+\tau-z)) .
$$

We have

$$
w(\tau)=H(t, x(t)), \quad w(t+\tau-u)=H(u, x(u))
$$

and hence,

$$
\begin{aligned}
w(t+\tau-u)-w(\tau) & \geq-\log \theta \int_{u}^{t} w(t+\tau-v) d v \\
& =\log (1 / \theta) \int_{\tau}^{t+\tau-u} w(z) d z
\end{aligned}
$$

Now, let $\alpha=t+\tau-u$. Since $u \in[\tau, t]$, we have $\alpha \in[\tau, t]$. Therefore,

$$
w(\alpha)-w(\tau) \geq \log (1 / \theta) \int_{\tau}^{\alpha} w(z) d z
$$

for $\alpha \in[\tau, t]$. Hence, it follows from Lemma 2.2 that $w(t) \geq \theta^{\tau-t} w(\tau)$, and so,

$$
H(t, x(t))=w(\tau) \leq \theta^{t-\tau} w(t)=\theta^{t-\tau} H(\tau, x)
$$

for $t \geq \tau$. This establishes inequality (2.9) when $[\tau, t]$ contains no jumping times. Otherwise, we proceed as for $F_{\tau}^{u}$. Indeed, it follows from equation (2.14) that

$$
H(t, x(t)) \leq \theta^{t-\tau_{i}} H\left(\tau_{i}^{+}, x\left(\tau_{i}^{+}\right)\right),
$$

and, since $t \mapsto H(t, x(t))$ is continuous, we have $H\left(\tau_{i}^{+}, x\left(\tau_{i}^{+}\right)\right)=$ $H\left(\tau_{i}, x\left(\tau_{i}\right)\right)$. Finally, on the interval $\left[\tau, \tau_{i}\right]$, it follows again from equation (2.14) that

$$
H\left(\tau_{i}, x\left(\tau_{i}\right)\right) \leq \theta^{\tau_{i}-\tau} H(\tau, x(\tau))
$$

and so,

$$
\begin{aligned}
H(t, x(t)) & \leq \theta^{t-\tau_{i}} H\left(\tau_{i}^{+}, x\left(\tau_{i}^{+}\right)\right)=\theta^{t-\tau_{i}} H\left(\tau_{i}, x\left(\tau_{i}\right)\right) \\
& \leq \theta^{t-\tau_{i}} \theta^{\tau_{i}-\tau} H(\tau, x(\tau))=\theta^{t-\tau} H(\tau, x),
\end{aligned}
$$

which establishes inequality (2.9). 
More generally, one can consider the situation when $t \mapsto H(t, x(t))$ is not continuous. The following is a generalization of Theorem 2.1 to this setting.

Theorem 2.3. Assume that $t \mapsto H(t, x(t))$ is in $\mathcal{D}$ for any solution $x(t)$ of equation (1.1). Moreover, assume that inequality (2.8) holds when $t$ is not a jumping time and there exists an $\eta>0$ such that

$$
H\left(\tau_{i}^{+},\left(\mathrm{Id}+B_{i}\right) x\right) \leq(1+\eta) H\left(\tau_{i}, x\right)
$$

for $i \in \mathbb{Z}$ and $x \in F_{\tau_{i}}^{s}$, and

$$
\left|H\left(\tau_{i}^{+},\left(\operatorname{Id}+B_{i}\right) x\right)\right| \geq(1-\eta)\left|H\left(\tau_{i}, x\right)\right|
$$

for $i \in \mathbb{Z}$ and $x \in F_{\tau_{i}}^{u}$. Then,

$$
H(t, T(t, \tau) x) \leq \theta^{t-\tau}(1+\eta)^{q(1+t-\tau)} H(\tau, x)
$$

for $t \geq \tau$ and $x \in F_{\tau}^{s}$, and

$$
|H(t, T(t, \tau) x)| \geq \theta^{-(t-\tau)}(1-\eta)^{q(1+t-\tau)}|H(\tau, x)|
$$

for $t \geq \tau$ and $x \in F_{\tau}^{u}$.

Proof. One can proceed as in the proof of Theorem 2.1 to show that

$$
|H(t, x(t))| \geq \theta^{-(t-\tau)}|H(\tau, x)|
$$

for any interval $[\tau, t]$ containing no jumping times and any $x \in F_{\tau}^{u}$.

Now we consider the case when $[\tau, t]$ contains a single jumping time. Proceeding as in equation (2.13), it follows from equation (2.17) that

$$
\begin{aligned}
|H(t, x(t))| & \geq \theta^{-\left(t-\tau_{i}\right)}\left|H\left(\tau_{i}^{+}, x\left(\tau_{i}^{+}\right)\right)\right| \\
& \geq \theta^{-\left(t-\tau_{i}\right)}(1-\eta)\left|H\left(\tau_{i}, x\left(\tau_{i}\right)\right)\right| \\
& \geq \theta^{-(t-\tau)}(1-\eta)|H(\tau, x(\tau))| .
\end{aligned}
$$

Finally, for $t \geq \tau$ and $x \in F_{\tau}^{u}$, by equation (2.1), we have

$$
\begin{aligned}
|H(t, x(t))| & \geq \theta^{-(t-\tau)}(1-\eta)^{\operatorname{card}\left\{i \in \mathbb{N}: \tau \leq \tau_{i}<t\right\}}|H(\tau, x(\tau))| \\
& \geq \theta^{-(t-\tau)}(1-\eta)^{q(1+t-\tau)}|H(\tau, x(\tau))| .
\end{aligned}
$$

Similarly,

$$
H(t, x(t)) \leq \theta^{(t-\tau)} H(\tau, x)
$$


for any interval $[\tau, t]$ containing no jumping times and any $x \in F_{\tau}^{s}$. When $[\tau, t]$ contains a single jumping time, proceeding as in equation (2.15) and using equation (2.16), we obtain

$$
\begin{aligned}
H(t, x(t)) & \leq \theta^{t-\tau_{i}} H\left(\tau_{i}^{+}, x\left(\tau_{i}^{+}\right)\right) \\
& \leq \theta^{t-\tau_{i}}(1+\eta) H\left(\tau_{i}, x\left(\tau_{i}\right)\right) \\
& \leq \theta^{t-s}(1+\eta) H(\tau, x(\tau)) .
\end{aligned}
$$

Finally, for $t \geq \tau$ and $x \in F_{\tau}^{s}$, it follows from equation (2.1) that

$$
\begin{aligned}
H(t, x(t)) & \leq \theta^{t-\tau}(1+\eta)^{\mathrm{card}\left\{i \in \mathbb{N}: \tau \leq \tau_{i}<t\right\}} H(\tau, x(\tau)) \\
& \leq \theta^{t-\tau}(1+\eta)^{q(1+t-\tau)} H(\tau, x(\tau)) .
\end{aligned}
$$

This concludes the proof of the theorem.

3. Characterization of exponential dichotomies. In this section, we give a characterization of the notion of an exponential dichotomy in terms of the functions $H$ and $\dot{H}$. This is used in Section 4 to prove the robustness property of a nonuniform exponential dichotomy.

We say that equation (1.1) admits a nonuniform exponential dichotomy if there exist projections $P(t)$ for $t \in \mathbb{R}$ satisfying

$$
T(t, s) P(s)=P(t) T(t, s), \quad t, s, \in \mathbb{R},
$$

and constants $a, b, D>0$ and $\varepsilon \geq 0$ such that

$$
\|T(t, s) P(s)\| \leq D e^{-a(t-s)+\varepsilon|s|}
$$

and

$$
\left\|T(t, s)^{-1} Q(t)\right\| \leq D e^{-b(t-s)+\varepsilon|t|}
$$

for $t \geq s$, where $Q(t)=\mathrm{Id}-P(t)$ for each $t \in \mathbb{R}$. The stable and unstable spaces at time $t$ are defined by

$$
E_{t}^{s}=P(t) \mathbb{R}^{p} \quad \text { and } \quad E_{t}^{u}=Q(t) \mathbb{R}^{p} .
$$

In the remainder of the paper we always assume that there exist constants $c>0$ and $\alpha, K \geq 0$, such that

$$
\|T(t, s)\| \leq K e^{\alpha|t|} \quad \text { for } \quad|t-s| \leq c .
$$


Given matrices $A, B \in M_{p}$ and a set $Y \subset \mathbb{R}^{p}$, we write $A|Y \leq B| Y$ if

$$
\langle A x, x\rangle \leq\langle B x, x\rangle \quad \text { for } \quad x \in Y .
$$

Moreover, we write $A \leq B$ if equation (3.2) holds with $Y=\mathbb{R}^{p}$.

The following two results give a characterization of the notion of an exponential dichotomy. We first give necessary conditions for the existence of a nonuniform exponential dichotomy.

Theorem 3.1. Assume that condition (3.1) holds. If equation (1.1) admits a nonuniform exponential dichotomy, then there exist symmetric invertible matrices $S(t)$ for $t \in \mathbb{R}$ and constants $L, \kappa>0$ such that:

(i) the function $t \mapsto S(t)$ is in $\mathcal{D}$ and, for each $t \in \mathbb{R}$, we have

$$
S(t) \leq L e^{2 \varepsilon|t|} \mathrm{Id}
$$

and

$$
S(t)\left|E_{t}^{s} \geq L^{-1} e^{-2(\alpha+\varepsilon)|t|} \mathrm{Id}, \quad-S(t)\right| E_{t}^{u} \geq L^{-1} e^{-2(\alpha+\varepsilon)|t|} \mathrm{Id}
$$

(ii) for each $t \in \mathbb{R}$ (including for $t=\tau_{i}^{+}$) and $x \in X$, we have

$$
\dot{H}(t, x) \leq-\kappa|H(t, x)| ;
$$

(iii) $t \mapsto H(t, x(t))$ is continuous for any solution $x(t)$ of equation (1.1), and so, in particular for each $i \in \mathbb{Z}$, we have

$$
\left(\operatorname{Id}+B_{i}\right)^{*} S\left(\tau_{i}^{+}\right)\left(\operatorname{Id}+B_{i}\right)=S\left(\tau_{i}\right) ;
$$

(iv) for every $t \in \mathbb{R}$ (including for $t=\tau_{i}^{+}$) and $i \in \mathbb{Z}$, we have

$$
S^{\prime}(t)+S(t) A(t)+A(t)^{*} S(t) \leq-\frac{1}{2} \operatorname{Id} .
$$

Proof. Given a positive number $\varrho<\min \{a, b\}$, we consider the matrices

$$
\begin{aligned}
S(t)= & \int_{t}^{\infty} T(v, t)^{*} P(v)^{*} P(v) T(v, t) e^{2(a-\varrho)(v-t)} d v \\
& -\int_{-\infty}^{t} T(v, t)^{*} Q(v)^{*} Q(v) T(v, t) e^{2(b-\varrho)(t-v)} d v .
\end{aligned}
$$


One can easily verify that $S(t)$ is symmetric for each $t \in \mathbb{R}$ and that the function $t \mapsto S(t)$ is in $\mathcal{D}$. Moreover, since $H(t, x)>0$ for $x \in E_{t}^{s} \backslash\{0\}$ and $H(t, x)<0$ for $x \in E_{t}^{u} \backslash\{0\}$, it follows readily from the identity $E_{t}^{s} \oplus E_{t}^{u}=\mathbb{R}^{p}$ that the matrix $S(t)$ is invertible for each $t$.

We observe that

$$
\begin{aligned}
\langle S(t) x, x\rangle= & \int_{t}^{\infty}\|T(v, t) P(t) x\|^{2} e^{2(a-\varrho)(v-t)} d v \\
& -\int_{-\infty}^{t}\|T(v, t) Q(t) x\|^{2} e^{2(b-\varrho)(t-v)} d v \\
\leq & D^{2} e^{2 \varepsilon|t|}\|x\|^{2}\left(\int_{t}^{\infty} e^{-2 \varrho(v-t)} d v+\int_{-\infty}^{t} e^{-2 \varrho(t-v)} d v\right) \\
= & \frac{D^{2}}{\varrho} e^{2 \varepsilon|t|}\|x\|^{2} .
\end{aligned}
$$

Moreover,

$$
\begin{aligned}
\langle S(t) x, x\rangle & \geq \int_{t}^{t+c}\|T(v, t) P(t) x\| e^{2(a-\varrho)(v-t)} d v \\
& \geq\|x\|^{2} \int_{t}^{t+c} \frac{1}{\|T(t, v) P(t)\|^{2}} e^{2(a-\varrho)(v-t)} d v \\
& \geq \frac{\|x\|^{2}}{K^{2} D^{2}} e^{-2 \alpha|t|-2 \varepsilon|t|} \int_{t}^{t+c} e^{2(a-\varrho)(v-t)} d v \\
& =\frac{e^{-2 \alpha|t|-2 \varepsilon|t|}\|x\|^{2}}{2 K^{2} D^{2}} \cdot \frac{e^{2(a-\varrho) c}-1}{a-\varrho}
\end{aligned}
$$

for $x \in E_{t}^{s}$ and

$$
\begin{aligned}
-\langle S(t) x, x\rangle & \geq \int_{t-c}^{t}\|T(v, t) Q(t) x\|^{2} e^{2(b-\varrho)(t-v)} d v \\
& \geq\|x\|^{2} \int_{t-c}^{t} \frac{1}{\|T(t, v) Q(t)\|^{2}} e^{2(b-\varrho)(t-v)} d v \\
& \geq \frac{\|x\|^{2}}{K^{2} D^{2}} e^{-2 \alpha|t|-2 \varepsilon|t|} \int_{t-c}^{t} e^{2(b-\varrho)(t-v)} \\
& =\frac{e^{-2 \alpha|t|-2 \varepsilon|t|}\|x\|^{2}}{2 K^{2} D^{2}} \cdot \frac{e^{2(b-\varrho) c}-1}{b-\varrho}
\end{aligned}
$$

for $x \in E_{t}^{u}$. This establishes inequalities (3.3) and (3.4). 
Furthermore, since

$$
\frac{\partial}{\partial t} T(\tau, t)=-T(\tau, t) A(t)
$$

and

$$
\frac{\partial}{\partial t} T(\tau, t)^{*}=-A(t)^{*} T(\tau, t)^{*}
$$

for $t$ outside the jumping times $\tau_{i}$, we obtain

$$
\begin{aligned}
S^{\prime}(t)= & -P(t)^{*} P(t) \\
& -\int_{t}^{\infty} A(t)^{*} T(v, t)^{*} P(v)^{*} P(v) T(v, t) e^{2(a-\varrho)(v-t)} d v \\
& -\int_{t}^{\infty} T(v, t)^{*} P(v)^{*} P(v) T(v, t) A(t) e^{2(a-\varrho)(v-t)} d v \\
& -2(a-\varrho) \int_{t}^{\infty} T(v, t)^{*} P(v)^{*} P(v) T(v, t) e^{2(a-\varrho)(v-t)} d v \\
& -Q(t)^{*} Q(t) \\
& +\int_{-\infty}^{t} A(t)^{*} T(v, t)^{*} Q(v)^{*} Q(v) T(v, t) e^{2(b-\varrho)(t-v)} d v \\
& +\int_{-\infty}^{t} T(v, t)^{*} Q(v)^{*} Q(v) T(v, t) A(t) e^{2(b-\varrho)(t-v)} d v \\
& -2(b-\varrho) \int_{-\infty}^{t} T(v, t)^{*} Q(v)^{*} Q(v) T(v, t) e^{2(b-\varrho)(t-v)} d v \\
= & -\left[P(t)^{*} P(t)+Q(t)^{*} Q(t)\right]-A(t)^{*} S(t)-S(t) A(t) \\
& -2(a-\varrho) \int_{t}^{\infty} T(v, t)^{*} P(v)^{*} P(v) T(v, t) e^{2(a-\varrho)(v-t)} d v \\
& -2(b-\varrho) \int_{-\infty}^{t} T(v, t)^{*} Q(v)^{*} Q(v) T(v, t) e^{2(b-\varrho)(t-v)} d v .
\end{aligned}
$$

On the other hand, for a solution $x(t)$ of equation (1.1), we have

$$
\begin{aligned}
\frac{d}{d t} H(t, x(t))= & \left\langle S^{\prime}(t) x(t), x(t)\right\rangle \\
& +\left\langle S(t) x^{\prime}(t), x(t)\right\rangle+\left\langle S(t) x(t), x^{\prime}(t)\right\rangle \\
= & \left\langle\left(S^{\prime}(t)+S(t) A(t)+A(t)^{*} S(t)\right) x(t), x(t)\right\rangle
\end{aligned}
$$


for $t \in \mathbb{R}$ (taking derivatives at $\tau_{i}$ and $\tau_{i}^{+}$, respectively, as in equations (2.4) and (2.5)). It follows from equation (3.10) that

$$
\begin{aligned}
\frac{d}{d t} H(t, x(t)) \leq & -\langle x(t), x(t)\rangle \\
& -2(a-\varrho) \int_{t}^{\infty}\|T(v, t) P(t) x(t)\|^{2} e^{2(a-\varrho)(v-t)} d v \\
& -2(b-\varrho) \int_{-\infty}^{t}\|T(v, t) Q(t) x(t)\|^{2} e^{2(b-\varrho)(t-v)} d v \\
\leq & -2(a-\varrho) \int_{t}^{\infty}\|T(v, t) P(t) x(t)\|^{2} e^{2(a-\varrho)(v-t)} d v \\
& -2(b-\varrho) \int_{-\infty}^{t}\|T(v, t) Q(t) x(t)\|^{2} e^{2(b-\varrho)(t-v)} d v .
\end{aligned}
$$

If $H(t, x(t)) \geq 0$, then by equation (3.11), and since $a-\varrho>0$ and $b-\varrho>0$, we obtain

$$
\begin{aligned}
\frac{d}{d t} H(t, x(t)) \leq & -2(a-\varrho) \int_{t}^{\infty}\|T(v, t) P(t) x(t)\|^{2} e^{2(a-\varrho)(v-t)} d v \\
& +2(a-\varrho) \int_{-\infty}^{t}\|T(v, t) Q(t) x(t)\|^{2} e^{2(b-\varrho)(t-v)} d v \\
= & -2(a-\varrho)|H(t, x(t))| .
\end{aligned}
$$

Analogously, if $H(t, x(t)) \leq 0$, then

$$
\begin{aligned}
\frac{d}{d t} H(t, x(t)) \leq & 2(b-\varrho) \int_{t}^{\infty}\|T(v, t) P(t) x(t)\|^{2} e^{2(a-\varrho)(v-t)} d v \\
& -2(b-\varrho) \int_{-\infty}^{t}\|T(v, t) Q(t) x(t)\|^{2} e^{2(b-\varrho)(t-v)} d v \\
= & -2(b-\varrho)|H(t, x(t))| .
\end{aligned}
$$

Taking $\kappa=\min \{a-\varrho, b-\varrho\}$, we obtain inequality (3.5).

For the continuity of the function $t \mapsto H(t, x(t))$, we note that $S\left(\tau_{i}\right)$ is given by

$$
\begin{aligned}
S\left(\tau_{i}\right)= & \int_{\tau_{i}}^{\infty} Y_{i}^{*} T\left(v, \tau_{i}^{+}\right)^{*} P(v)^{*} P(v) T\left(v, \tau_{i}^{+}\right) Y_{i} e^{2(a-\varrho)(v-t)} d v \\
& -\int_{-\infty}^{\tau_{i}} Y_{i}^{*} T\left(v, \tau_{i}^{+}\right)^{*} Q(v)^{*} Q(v) T\left(v, \tau_{i}^{+}\right) Y_{i} e^{2(b-\varrho)(t-v)} d v
\end{aligned}
$$




$$
=\left(\mathrm{Id}+B_{i}\right)^{*} S\left(\tau_{i}^{+}\right)\left(\mathrm{Id}+B_{i}\right),
$$

where $Y_{i}=\mathrm{Id}+B_{i}$, and so,

$$
\begin{aligned}
& H\left(\tau_{i}^{+}, x\left(\tau_{i}^{+}\right)\right)-H\left(\tau_{i}, x\left(\tau_{i}\right)\right) \\
& =\left\langle S\left(\tau_{i}^{+}\right)\left(\operatorname{Id}+B_{i}\right) x\left(\tau_{i}\right),\left(\operatorname{Id}+B_{i}\right) x\left(\tau_{i}\right)\right\rangle-\left\langle S\left(\tau_{i}\right) x\left(\tau_{i}\right), x\left(\tau_{i}\right)\right\rangle \\
& =\left\langle\left(\operatorname{Id}+B_{i}\right)^{*} S\left(\tau_{i}^{+}\right)\left(\operatorname{Id}+B_{i}\right) x\left(\tau_{i}\right), x\left(\tau_{i}\right)\right\rangle-\left\langle S\left(\tau_{i}\right) x\left(\tau_{i}\right), x\left(\tau_{i}\right)\right\rangle \\
& =\left\langle\left[\left(\operatorname{Id}+B_{i}\right)^{*} S\left(\tau_{i}^{+}\right)\left(\operatorname{Id}+B_{i}\right)-S\left(\tau_{i}\right)\right] x\left(\tau_{i}\right), x\left(\tau_{i}\right)\right\rangle=0 .
\end{aligned}
$$

This establishes the continuity of the function $t \mapsto H(t, x(t))$ at the jumping times. For the other times, since $t \mapsto x(t)$ is continuous outside the jumping times and $H(t, x(t))=\langle S(t) x(t), x(t)\rangle$, it is sufficient to recall that $t \mapsto S(t)$ is in $\mathcal{D}$.

Finally, since $a-\varrho>0$ and $b-\varrho>0$, it follows from equation (3.10) that

$$
\begin{aligned}
& \left\langle\left(S^{\prime}(t)+S(t) A(t)+A(t)^{*} S(t)+P(t)^{*} P(t)+Q(t)^{*} Q(t)\right) x, x\right\rangle \\
& =-2(a-\varrho) \int_{t}^{\infty}\|T(v, t) P(t) x\|^{2} e^{-2(a+\varrho)(v-t)} d v \\
& \quad-2(b-\varrho) \int_{-\infty}^{t}\|T(v, t) Q(t) x\|^{2} e^{2(b-\varrho)(t-v)} d v \leq 0
\end{aligned}
$$

On the other hand,

$$
\begin{aligned}
2\left\langle\left(P(t)^{*} P(t)+Q(t)^{*} Q(t)\right)\right\rangle= & 2\|P(t) x\|^{2}+2\|Q(t) x\|^{2} \\
\geq & \|P(t) x\|^{2}+\|Q(t) x\|^{2} \\
& +2\|P(t) x\| \cdot\|Q(t) x\| \\
= & (\|P(t) x\|+\|Q(t) x\|)^{2} \\
\geq & \|(P(t)+Q(t)) x\|^{2} \\
= & \|x\|^{2}
\end{aligned}
$$

and hence,

$$
P(t)^{*} P(t)+Q(t)^{*} Q(t) \geq \frac{1}{2} \mathrm{Id} .
$$

Together with equation (3.13), this yields inequality (3.7).

Now, we consider the other direction and give sufficient conditions for the existence of a nonuniform exponential dichotomy. Recall the sets $F_{t}^{s}$ and $F_{t}^{u}$ introduced in equations (2.6) and (2.7). 
Theorem 3.2. If there exist symmetric invertible linear operators $S(t)$ for $t \in \mathbb{R}$ and constants $L, \kappa>0$ and $\varepsilon \geq 0$ satisfying Theorem 3.1 (i)-(iii) with $E_{t}^{s}$ and $E_{t}^{u}$ replaced, respectively, by $F_{t}^{s}$ and $F_{t}^{u}$ and with $\kappa>2(\varepsilon+\alpha)$, then equation (1.1) admits a nonuniform exponential dichotomy.

Proof. We begin with two auxiliary lemmas.

Lemma 3.3. For every $t, \tau \in \mathbb{R}$ with $t \geq \tau$, we have

$$
\left\|T(t, \tau) \mid F_{\tau}^{s}\right\|^{2} \leq L^{2} e^{-(\kappa-2 \varepsilon-2 \alpha)(t-\tau)+2(2 \varepsilon+\alpha)|\tau|}
$$

and

$$
\left\|T(t, \tau)^{-1} \mid F_{t}^{u}\right\|^{2} \leq L^{2} e^{-(\kappa-2 \varepsilon-2 \alpha)(t-\tau)+2(2 \varepsilon+\alpha)|t|} .
$$

Proof of Lemma 3.3. For $x \in F_{\tau}^{s}$ and $t \geq \tau$, we have $T(t, \tau) x \in F_{t}^{s}$, and so, by conditions (i)-(ii) of Theorems 2.1 and 3.1 ,

$$
\begin{aligned}
\|T(t, \tau) x\|^{2} & \leq L e^{2(\varepsilon+\alpha)|t|} H(t, T(t, \tau) x) \\
& \leq L e^{2(\varepsilon+\alpha)|t|} e^{-\kappa(t-\tau)} H(\tau, x) \\
& \leq L^{2} e^{2(\varepsilon+\alpha)|t|+2 \varepsilon|\tau|} e^{-\kappa(t-\tau)}\|x\|^{2} \\
& \leq L^{2} e^{2(2 \varepsilon+\alpha)|\tau|} e^{-(\kappa-2 \varepsilon-2 \alpha)(t-\tau)}\|x\|^{2} .
\end{aligned}
$$

Similarly, for $x \in F_{\tau}^{u}$ and $t \geq \tau$, we have $T(t, \tau) x \in F_{t}^{u}$, and so,

$$
\begin{aligned}
\|T(t, \tau) x\|^{2} & \geq L^{-1} e^{-2 \varepsilon|t|}|H(t, T(t, \tau) x)| \\
& \geq L^{-1} e^{-2 \varepsilon|t|} e^{\kappa(t-\tau)}|H(\tau, x)| \\
& \geq L^{-2} e^{-2 \varepsilon|t|-2(\varepsilon+\alpha)|\tau|} e^{\kappa(t-\tau)}\|x\|^{2} \\
& \geq L^{-2} e^{-2(2 \varepsilon+\alpha)|t|} e^{(\kappa-2 \varepsilon-2 \alpha)(t-\tau)}\|x\|^{2} .
\end{aligned}
$$

This completes the proof of Lemma 3.3.

Lemma 3.4. For each $t \in \mathbb{R}$ the sets $F_{t}^{s}$ and $F_{t}^{u}$ are subspaces and form the direct sum $F_{t}^{s} \oplus F_{t}^{u}=\mathbb{R}^{p}$.

Proof of Lemma 3.4. For each $t, \tau \in \mathbb{R}$, let

$$
C_{t, \tau}^{u}=\left\{T(t, \tau) x \in \mathbb{R}^{p}: H(\tau, x) \leq 0\right\}
$$


and

$$
C_{t, \tau}^{s}=\left\{T(t, \tau) x \in \mathbb{R}^{p}: H(\tau, x) \geq 0\right\} .
$$

By Theorem 3.1 (ii), we have $\dot{H}(t, x) \leq 0$, and so,

$$
H(t, T(t, \tau) x) \leq H(\tau, x)
$$

for every $t \geq \tau$ and $x \in \mathbb{R}^{p}$. Therefore,

$$
C_{t, \tau_{1}}^{u} \supset C_{t, \tau_{2}}^{u} \quad \text { and } \quad C_{t, \tau_{2}}^{s} \supset C_{t, \tau_{1}}^{s}
$$

for every $t, \tau_{1}, \tau_{2} \in \mathbb{R}$ with $\tau_{1} \geq \tau_{2}$. Since the matrices $S(t)$ have constant index, there exist integers $r_{s}, r_{u} \geq 0$ with $r_{s}+r_{u}=p$ such that each set $C_{t, \tau}^{u}$ contains a subspace of dimension $r_{u}$ and each set $C_{t, \tau}^{s}$ a subspace of dimension $r_{s}$. Hence, it follows from equation (3.14) and the compactness of the closed unit ball in $\mathbb{R}^{p}$ that the intersections

$$
D_{t}^{u}=\bigcap_{\tau \in \mathbb{R}} C_{t, \tau}^{u} \quad \text { and } \quad D_{t}^{s}=\bigcap_{\tau \in \mathbb{R}} C_{t, \tau}^{s}
$$

contain subspaces, respectively, of dimensions $r_{u}$ and $r_{s}$. On the other hand, one can easily verify that, for each $t \in \mathbb{R}$,

$$
D_{t}^{u}=F_{t}^{u} \quad \text { and } \quad D_{t}^{s}=F_{t}^{s} .
$$

Now let $E_{t}^{u} \subset D_{t}^{u}$ be any $r_{u}$-dimensional subspace, and let $E_{t}^{s} \subset D_{t}^{s}$ be any $r_{s}$-dimensional subspace. By Lemma 3.3, we have

$$
E_{t}^{u} \cap E_{t}^{s} \subset F_{t}^{u} \cap F_{t}^{s}=\{0\} .
$$

In order to show that $E_{t}^{s}=D_{t}^{s}$, we assume that there exists a vector $x$ in $D_{t}^{s}$ that is not in $E_{t}^{s}$. Then $x$ would have a component in $E_{t}^{u}$, and so the solution starting at $x$ would contract and expand simultaneously as time grows, which is impossible. Hence, $D_{t}^{s}=E_{t}^{s}$. Since $E_{t}^{s}$ is a vector space, we have that $F_{t}^{s}=D_{t}^{s}=E_{t}^{s}$ is also a vector space. In a similar manner, we can show that $F_{t}^{u}=D_{t}^{u}=E_{t}^{u}$ and thus $F_{t}^{u}$ is a vector space. This completes the proof of the lemma.

Now let

$$
P(t): \mathbb{R}^{p} \rightarrow F_{t}^{s} \quad \text { and } \quad Q(t): \mathbb{R}^{p} \rightarrow F_{t}^{u}
$$

be the projections associated to the decomposition $F_{t}^{s} \oplus F_{t}^{u}=\mathbb{R}^{p}$. 
Lemma 3.5. We have

$$
\|P(t)\|=\|Q(t)\| \leq \sqrt{2} L e^{2(\alpha+\varepsilon)|t|}\|S(t)\|, \quad t \in \mathbb{R} .
$$

Proof of Lemma 3.5. Given $x \in \mathbb{R}^{p}$, we write it in the form $x=y+z$ with $y \in F_{t}^{s}$ and $z \in F_{t}^{u}$. We have $H(t, y) \geq 0$ and $H(t, z) \leq 0$. Take $a(t)>0$, and let

$$
H^{s}(t, y)=-\langle S(t) y, y\rangle+a(t)\|y\|^{2} .
$$

By equation (3.4), we have

$$
\begin{aligned}
H^{s}(t, y) & \leq-\frac{1}{L} e^{-2(\alpha+\varepsilon)|t|}\|y\|^{2}+a(t)\|y\|^{2} \\
& =\left(a(t)-\frac{1}{L} e^{-2(\alpha+\varepsilon)|t|}\right)\|y\|^{2} .
\end{aligned}
$$

Similarly, let

$$
H^{u}(t, z)=-\langle S(t) z, z\rangle-a(t)\|z\|^{2} .
$$

Again, by equation (3.4), we have

$$
H^{u}(t, z) \geq\left(\frac{1}{L} e^{-2(\alpha+\varepsilon)|t|}-a(t)\right)\|z\|^{2} .
$$

Hence, if $a(t) \leq e^{-2(\alpha+\varepsilon)|t|} / L$, then

$$
-H(t, y)+a(t)\|y\|^{2} \leq 0 \quad \text { and } \quad-H(t, z)-a(t)\|z\|^{2} \geq 0 .
$$

Since $S(t)$ is symmetric, subtracting the two inequalities, we obtain

$$
\begin{aligned}
0 \geq & a(t)\|P(t) x\|^{2}+a(t)\|Q(t) x\|^{2} \\
& -\langle S(t) P(t) x, P(t) x\rangle+\langle S(t) Q(t) x, Q(t) x\rangle \\
= & a(t)\|P(t) x\|^{2}+a(t)\|Q(t) x\|^{2}+\langle S(t) x, x\rangle-2\langle S(t) P(t) x, x\rangle .
\end{aligned}
$$

Therefore,

$$
\begin{aligned}
a(t) \| & P(t) x-\frac{1}{2 a(t)} S(t) x\left\|^{2}+a(t)\right\| Q(t) x+\frac{1}{2 a(t)} S(t) x \|^{2} \\
= & a(t)\|P(t) x\|^{2}+a(t)\|Q(t) x\|^{2}+\frac{\|S(t) x\|^{2}}{2 a(t)} \\
& +\langle S(t) x, x\rangle-2\langle S(t) P(t) x, x\rangle \leq \frac{\|S(t) x\|^{2}}{2 a(t)}
\end{aligned}
$$


and

$$
\left\|P(t) x-\frac{1}{2 a(t)} S(t) x\right\|^{2}+\left\|Q(t) x+\frac{1}{2 a(t)} S(t) x\right\|^{2} \leq \frac{\|S(t) x\|^{2}}{2 a(t)^{2}} .
$$

This implies that

$$
\begin{aligned}
\|P(t) x\| & =\left\|P(t) x-\frac{1}{2 a(t)} S(t) x+\frac{1}{2 a(t)} S(t) x\right\| \\
& \leq\left\|P(t) x-\frac{1}{2 a(t)} S(t) x\right\|+\frac{1}{2 a(t)}\|S(t) x\| \\
& \leq \frac{1}{\sqrt{2} a(t)}\|S(t) x\|+\frac{1}{2 a(t)}\|S(t) x\| \\
& \leq \frac{\sqrt{2}}{a(t)}\|S(t) x\|
\end{aligned}
$$

and, similarly,

$$
\begin{aligned}
\|Q(t) x\| & \leq\left\|Q(t) x+\frac{1}{2 a(t)} S(t) x-\frac{1}{2 a(t)} S(t) x\right\| \\
& \leq\left\|Q(t) x+\frac{1}{2 a(t)} S(t) x\right\|+\frac{1}{2 a(t)}\|S(t) x\| \\
& \leq \frac{1}{\sqrt{2} a(t)}\|S(t) x\|+\frac{1}{2 a(t)}\|S(t) x\| \\
& \leq \frac{\sqrt{2}}{a(t)}\|S(t) x\| .
\end{aligned}
$$

Taking $a(t)=e^{-2(\alpha+\varepsilon)|t|} / L$, we obtain the desired statement.

Finally, we observe that

$$
\|T(t, \tau) P(\tau)\| \leq\left\|T(t, \tau) \mid F_{\tau}^{s}\right\| \cdot\|P(\tau)\|
$$

and

$$
\left\|T(t, \tau)^{-1} Q(t)\right\| \leq\left\|T(t, \tau)^{-1} \mid F_{t}^{u}\right\| \cdot\|Q(t)\|
$$

for $t \geq \tau$, and so, it follows from Lemmas 3.3 and 3.5 that equation (1.1) admits a nonuniform exponential dichotomy.

The following result is a version of Theorem 3.2 when Theorem 3.1 (iii) does not hold (we also do not require condition (3.1)). 
Theorem 3.6. If there exist symmetric invertible linear operators $S(t)$ for $t \in \mathbb{R}$ and constants $L, \kappa>0$ and $\alpha, \varepsilon \geq 0$ satisfying Theorem 3.1 (i)-(ii), and

$$
\left(\mathrm{Id}+B_{i}\right)^{*} S\left(\tau_{i}^{+}\right)\left(\mathrm{Id}+B_{i}\right)-S\left(\tau_{i}\right) \leq \eta S\left(\tau_{i}\right)
$$

for $i \in \mathbb{Z}$ and some $\eta \in(0,1)$ with

$$
\kappa>2(\varepsilon+\alpha)+q \log (1+\eta),
$$

then equation (1.1) admits a nonuniform exponential dichotomy.

Proof. We first prove an auxiliary result.

Lemma 3.7. For every $t, \tau \in \mathbb{R}$ with $t \geq \tau$, we have

$$
\left\|T(t, \tau) \mid F_{\tau}^{s}\right\|^{2} \leq L^{2}(1+\eta)^{q} e^{2(2 \varepsilon+\alpha)|\tau|} e^{-(\kappa-2 \varepsilon-2 \alpha-q \log (1+\eta))(t-\tau)},
$$

and

$$
\left\|T(t, \tau)^{-1} \mid F_{t}^{u}\right\|^{2} \leq(1-\eta)^{-q} L^{2} e^{2(2 \varepsilon+\alpha)|t|} e^{-(\kappa-2 \varepsilon-2 \alpha-q \log (1-\eta))(t-\tau)} .
$$

Proof of Lemma 3.7. If $x \in F_{\tau}^{s}$ and $t \geq \tau$, then, in view of equations (3.4), (3.5) and Theorem 2.3, we have

$$
\begin{aligned}
\|T(t, \tau) x\|^{2} & \leq L e^{2(\varepsilon+\alpha)|t|} H(t, T(t, \tau) x) \\
& \leq L e^{2(\varepsilon+\alpha)|t|} e^{-\kappa(t-\tau)}(1+\eta)^{q(1+t-\tau)} H(\tau, x) \\
& \leq L^{2} e^{2(\varepsilon+\alpha)|t|+2 \varepsilon|\tau|} e^{-\kappa(t-\tau)}(1+\eta)^{q(1+t-\tau)}\|x\|^{2} \\
& \leq L^{2}(1+\eta)^{q} e^{2(2 \varepsilon+\alpha)|\tau|} e^{-(\kappa-2 \varepsilon-2 \alpha-q \log (1+\eta))(t-\tau)}\|x\|^{2} .
\end{aligned}
$$

Similarly, for $x \in F_{\tau}^{u}$ and $t \geq \tau$, we have

$$
\begin{aligned}
\|T(t, \tau) x\|^{2} & \geq L^{-1} e^{-2 \varepsilon|t|}|H(t, T(t, \tau) x)| \\
& \geq L^{-1} e^{-2 \varepsilon|t|} e^{\kappa(t-\tau)}(1-\eta)^{q(1+t-\tau)}|H(\tau, x)| \\
& \geq L^{-2} e^{-2 \varepsilon|t|-2(\varepsilon+\alpha)|\tau|} e^{\kappa(t-\tau)}(1-\eta)^{q(1+t-\tau)}\|x\|^{2} \\
& \geq(1-\eta)^{q} L^{-2} e^{-2(2 \varepsilon+\alpha)|t|} e^{(\kappa-2 \varepsilon-2 \alpha-q \log (1-\eta))(t-\tau)}\|x\|^{2} .
\end{aligned}
$$

This completes the proof of Lemma 3.7. 
We note that the statements in Lemmas 3.4 and 3.5 also hold in this setting. Thus, proceeding as in the proof of Theorem 3.2, we conclude that equation (1.1) admits a nonuniform exponential dichotomy.

4. Robustness of nonuniform exponential dichotomies. In this section, we establish the robustness of a nonuniform exponential dichotomy as an application of the results in the former sections. This means that, for any sufficiently small perturbation (in some appropriate sense) equation (1.2) still admits a nonuniform exponential dichotomy.

Theorem 4.1. Let $A, B: \mathbb{R} \rightarrow B(X)$ be continuous functions, and let $B_{i}, C_{i} \in B(X)$ be linear operators for $i \in \mathbb{Z}$ such that equation (1.1) admits a nonuniform exponential dichotomy. If condition (3.1) holds with $\alpha \leq \varepsilon$ and

$$
\|B(t)\| \leq \mu e^{-2 \varepsilon|t|}, \quad\left\|C_{i}\right\| \leq \mu e^{-7 \varepsilon\left|\tau_{i}\right|}
$$

for $t \in \mathbb{R}$ and $i \in \mathbb{Z}$ with $\mu$ sufficiently small, then equation (1.2) also admits a nonuniform exponential dichotomy.

Proof. Consider the matrices $S(t)$ in equation (3.8). Clearly, Theorem 3.1 (i) holds. Now we show that condition (ii) and equation (3.15) also hold when the dynamics of equation (1.1) is replaced by that of equation (1.2). If $x(t)$ is a solution of equation (1.2), then

$$
\begin{aligned}
\frac{d}{d t} H(t, x(t))= & \left\langle S^{\prime}(t) x(t), x(t)\right\rangle \\
& +\langle S(t) A(t) x(t), x(t)\rangle+\langle S(t) B(t) x(t), x(t)\rangle \\
& +\left\langle A(t)^{*} S(t) x(t), x(t)\right\rangle+\left\langle B(t)^{*} S(t) x(t), x(t)\right\rangle .
\end{aligned}
$$

By equation (3.10), we obtain

$$
\begin{aligned}
& \left\langle S^{\prime}(t) x(t), x(t)\right\rangle+\langle S(t) A(t) x(t), x(t)\rangle+\left\langle A(t)^{*} S(t) x(t), x(t)\right\rangle \\
& =-\frac{1}{2}\|x(t)\|^{2}-2(a-\varrho) \int_{t}^{\infty}\|T(v, t) P(t) x(t)\|^{2} e^{2(a-\varrho)(v-t)} d v \\
& \quad-2(b-\varrho) \int_{-\infty}^{t}\|T(v, t) Q(t) x(t)\|^{2} e^{2(b-\varrho)(t-v)} d v .
\end{aligned}
$$

Moreover, by equations (3.3) and (4.1), we have

$$
\langle S(t) B(t) x(t), x(t)\rangle+\left\langle B(t)^{*} S(t) x(t), x(t)\right\rangle \leq 2 L \mu\|x(t)\|^{2} .
$$


Therefore, it follows from equations (4.2) and (4.3) that, if $\mu$ is such that $2 L \mu<1 / 2$, then

$$
\begin{aligned}
& \frac{d}{d t} H(t, x(t)) \\
& \leq-2(a-\varrho) \int_{t}^{\infty}\|T(v, t) P(t) x(t)\|^{2} e^{2(a-\varrho)(v-t)} d v \\
& \quad-2(b-\varrho) \int_{-\infty}^{t}\|T(v, t) Q(t) x(t)\|^{2} e^{2(b-\varrho)(t-v)} d v
\end{aligned}
$$

Hence, if $H(t, x(t)) \geq 0$, then by equation (4.4) and in a similar manner to that in equation $(3.11)$, since $a-\varrho>0$ and $b-\varrho>0$, we obtain

$$
\frac{d}{d t} H(t, x(t)) \leq-2(a-\varrho)|H(t, x(t))| .
$$

Analogously, if $H(t, x(t)) \leq 0$, then

$$
\frac{d}{d t} H(t, x(t)) \leq-2(b-\varrho)|H(t, x(t))| .
$$

Thus, taking $\kappa=\min \{a-\varrho, b-\varrho\}$, we obtain Theorem 3.1 (ii).

Moreover, it follows from equation (3.6) that

$$
\begin{aligned}
J:= & \left(\mathrm{Id}+B_{i}+C_{i}\right)^{*} S\left(\tau_{i}^{+}\right)\left(\operatorname{Id}+B_{i}+C_{i}\right)-S\left(\tau_{i}\right) \\
= & \left(\operatorname{Id}+B_{i}+C_{i}\right)^{*} S\left(\tau_{i}^{+}\right)\left(\operatorname{Id}+B_{i}+C_{i}\right) \\
& -\left(\operatorname{Id}+B_{i}\right)^{*} S\left(\tau_{i}^{+}\right)\left(\operatorname{Id}+B_{i}\right) \\
= & \left(\operatorname{Id}+B_{i}\right)^{*} S\left(\tau_{i}\right) C_{i}+C_{i}^{*} S\left(\tau_{i}\right)\left(\mathrm{Id}+B_{i}+C_{i}\right) .
\end{aligned}
$$

Since

$$
\left\|\mathrm{Id}+B_{i}\right\|=\left\|T\left(\tau_{i}^{+}, \tau_{i}\right)\right\| \leq K e^{\varepsilon\left|\tau_{i}\right|},
$$

it follows from equation (4.5) that

$$
\begin{aligned}
\|J\| & \leq 2\left\|C_{i}\right\| \cdot\left\|S\left(\tau_{i}\right)\right\| \cdot\left(\left\|\mathrm{Id}+B_{i}\right\|+\left\|C_{i}\right\|\right) \\
& \leq 2 \mu(K+\mu) e^{-6 \varepsilon\left|\tau_{i}\right|}\left\|S\left(\tau_{i}\right)\right\| .
\end{aligned}
$$

On the other hand, since $S(t)$ is symmetric, by equation (3.3), we have

$$
\|S(t)\|=\max _{\|x\|=1}\langle S(t) x, x\rangle \leq L e^{2 \varepsilon|t|} .
$$

Therefore,

$$
\|J\| \leq 2 \mu(K+\mu) L e^{-4 \varepsilon\left|\tau_{i}\right|}
$$


and so, by equation (3.9),

$$
\begin{aligned}
\langle J x, x\rangle & \leq\|J\| \cdot\|x\|^{2} \\
& \leq 2 \mu(K+\mu) L^{2} e^{-4 \varepsilon\left|\tau_{i}\right|} e^{2(\alpha+\varepsilon)\left|\tau_{i}\right|}\left\langle S\left(\tau_{i}\right) x, x\right\rangle \\
& \leq 2 \mu(K+\mu) L^{2}\left\langle S\left(\tau_{i}\right) x, x\right\rangle .
\end{aligned}
$$

Hence, condition (3.15) holds for any sufficiently small $\mu$. Applying Theorem 3.6 yields that equation (1.2) admits a nonuniform exponential dichotomy.

\section{REFERENCES}

1. L. Barreira and Ya. Pesin, Nonuniform hyperbolicity, in Encyclopedia of mathematics and its applications, 115, Cambridge University Press, Cambridge, 2007.

2. L. Barreira and C. Valls, Quadratic Lyapunov functions and nonuniform exponential dichotomies, J. Differ. Equat. 246 (2009), 1235-1263.

3. , Robustness for impulsive equations, Nonlin. Anal. 72 (2010), 25422563.

4. N. Bhatia and G. Szegö, Stability theory of dynamical systems, Grundl. Math. Wissen. 161, Springer, Berlin, 1970.

5. S.-N. Chow and H. Leiva, Existence and roughness of the exponential dichotomy for skew-product semiflow in Banach spaces, J. Differ. Equat. 120 (1995), 429-477.

6. W. Coppel, Dichotomies and reducibility, J. Differ. Equat. 3 (1967), 500-521.

7. Ju. Dalec'kiı̆ and M. Krěn, Stability of solutions of differential equations in Banach space, Trans. Math. Mono. 43, American Mathematical Society, Providence, 1974.

8. W. Hahn, Stability of motion, Grundl. Math. Wissen. 138, Springer, Berlin, 1967.

9. V. Lakshmikantham, D. Bainov and P. Simeonov, Theory of impulsive differential equations, Ser. Modern Appl. Math. 6, World Scientific, Singapore, 1989.

10. J. LaSalle and S. Lefschetz, Stability by Liapunov's direct method, with applications, Math. Sci. Eng. 4, Academic Press, New York, 1961.

11. A. Lyapunov, The general problem of the stability of motion, Taylor \& Francis, London, 1992.

12. J. Massera and J. Schäffer, Linear differential equations and functional analysis, I, Ann. Math. 67 (1958), 517-573.

13. Linear differential equations and function spaces, Pure Appl. Math. 21, Academic Press, New York, 1966.

14. R. Naulin and M. Pinto, Admissible perturbations of exponential dichotomy roughness, Nonlin. Anal. 31 (1998), 559-571. 
15. V. Oseledets, A multiplicative ergodic theorem. Liapunov characteristic numbers for dynamical systems, Trans. Moscow Math. Soc. 19 (1968), 197-221.

16. Ya. Pesin, Families of invariant manifolds corresponding to nonzero characteristic exponents, Math. USSR-Izv. 10 (1976), 1261-1305.

17. V. Pliss and G. Sell, Robustness of exponential dichotomies in infinitedimensional dynamical systems, J. Dynam. Diff. Eq. 11 (1999), 471-513.

18. L. Popescu, Exponential dichotomy roughness on Banach spaces, J. Math. Anal. Appl. 314 (2006), 436-454.

19. A. Samoilenko and N. Perestyuk, Impulsive differential equations, Nonlin. Sci. Mono. Treatises 14, World Scientific, Singapore, 1995.

Departamento de Matemática, Instituto Superior Técnico, Universidade De Lisboa, 1049-001 Lisboa, Portugal

Email address: barreira@math.ist.utl.pt

Departamento de Matemática, Instituto Superior Técnico, Universidade De LisboA, 1049-001 LisboA, Portugal

Email address: cvalls@math.ist.utl.pt 